Recepción: 27 / 11 / 2017

Aceptación: 18 / 01 / 2018

Publicación: 21 / 03 / 2018

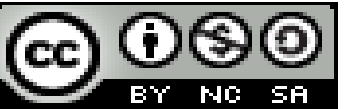

Ciencias económicas y empresariales

Artículo de Revisión

\title{
Inbound marketing como estrategia de fidelización de clientes
}

\author{
Inbound marketing as a customer loyalty strategy
}

\section{Inbound marketing como estratégia de fidelização de clientes}

\author{
Mauricio S. Quiroga-López ${ }^{\text {I }}$ \\ mauricioquiroga@hotmail.com \\ Karen G. Pinargote-Montenegro II \\ kgpinargote@hotmail.com
}

Correspondencia: mauricioquiroga@hotmail.com

\footnotetext{
${ }^{\mathrm{I}}$ Magister en Gerencia Empresarial (MBA) Mención Gestión de Proyectos, Abogado, Ingeniero Industrial, Docente de la Universidad Técnica de Ambato, Ambato; Ecuador.

${ }^{\text {II }}$ Magister en Administración De Empresas (MBA) Programa Integral de Habilidades Múltiples, Ingeniera Comercial, Universidad Laica Eloy Alfaro de Manabí, Manta, Ecuador.
} 


\section{Resumen}

Hoy día, las estrategias de fidelización; que en términos sencillos podría entenderse como el conjunto de medidas administrativas encomendadas por determinada gerencia con el objetivo de generar en sus actuales y potenciales clientes una preferencia o fidelidad hacia su propia marca, producto o servicio, representan un tema fundamental que los directivos empresariales toman en cuenta al momento de la planificación estratégica vinculada, por ejemplo, al posicionamiento o reposicionamiento de sus marcas, el aumento del volumen de sus ventas, mejoramiento de la productividad o cualquier otro aspecto considerados de valor en las organizaciones que dirigen. Precisamente es en esos aspectos donde puede afirmarse que el Inbound marketing juega cada vez más juega un papel protagónico entre las distintas estrategias de fidelización de clientes. Fue dentro de ese contexto que surgió la idea de desarrollar este tema, delimitando su desarrollo al de un trabajo investigativo de naturaleza documental y con una profundidad a nivel descriptivo, con el que se logró exponer sobre algunos de los métodos de fidelización más utilizados en la actualidad, haciendo especial énfasis en la descripción del Inbound marketing, todo ello con la finalidad de proporcionar un material que sirva tanto de ampliación de conocimientos para entendidos en la materia como de fundamento teórico en ulteriores trabajos de investigación relacionados con la fidelización de clientes. Finalmente se pudo concluir que, por su fácil aplicación y relativos bajos costos de ejecución, el Inbound marketing podría ser considerado en la actualidad como una excelente alternativa en comparación con otros métodos de fidelización de clientes.

Palabras clave: medidas; gerencia; clientes; estrategias; fidelización.

\section{Abstract}

Nowadays, loyalty strategies; that in simple terms could be understood as the set of administrative measures entrusted by certain management in order to generate in their current and potential customers a preference or loyalty to their own brand, product or service, represent a fundamental issue that business executives take in account at the time of strategic planning linked, for example, to the positioning or repositioning of their brands, the increase in the volume of their sales, improvement of productivity or any other aspect considered valuable in the organizations they manage. It is precisely in those aspects where it can be affirmed that inbound marketing plays more and more plays a leading role among the different strategies of customer 
loyalty. It was within this context that the idea of developing this theme arose, delimiting its development to that of a documentary work of documentary nature and with a depth at a descriptive level, with which it was possible to expose some of the most used loyalty methods in the Currently, with special emphasis on the description of inbound marketing, all with the aim of providing a material that serves both to expand knowledge for experts in the field and theoretical basis in further research related to customer loyalty. Finally, it could be concluded that, due to its easy application and relative low execution costs, inbound marketing could be considered an excellent alternative in comparison with other methods of customer loyalty.

Keywords: measurements; management; customers; strategies; loyalty.

\section{Resumo}

Atualmente, estratégias de fidelização; que, em termos simples, poderia ser entendido como o conjunto de medidas administrativas confiadas por determinada administração a fim de gerar em seus clientes atuais e potenciais uma preferência ou lealdade à sua própria marca, produto ou serviço, representam uma questão fundamental que os executivos tomam. Conta no momento do planejamento estratégico atrelado, por exemplo, ao posicionamento ou reposicionamento de suas marcas, ao aumento no volume de suas vendas, melhoria de produtividade ou qualquer outro aspecto considerado valioso nas organizações que gerenciam. É precisamente nesses aspectos que se pode afirmar que o marketing de entrada desempenha um papel cada vez mais importante entre as diferentes estratégias de fidelização de clientes. Foi dentro desse contexto que a ideia de desenvolver este tema, limitando o seu desenvolvimento para o trabalho de investigação de natureza documental e um nível descritivo profundidade, com o qual foi possível expor um pouco da lealdade métodos mais utilizados Atualmente, com especial ênfase na descrição do Inbound marketing, tudo com o objetivo de fornecer um material que sirva tanto para ampliar o conhecimento para especialistas no campo e base teórica em pesquisas futuras relacionadas à fidelidade do cliente. Finalmente, pode-se concluir que, devido à sua fácil aplicação e baixos custos de execução, o Inbound marketing pode ser considerado uma excelente alternativa em comparação com outros métodos de fidelização de clientes.

Palavras chave: medidas; gestão; clientes; estratégias; lealdade. 


\section{Introducción}

En vista de que en la actualidad la competencia entre empresas se hace cada vez más reñida debido a las pretensiones que éstas siempre tienen por dominar una mayor parte del mercado, podría afirmarse que cualquiera de ellas, bien sea ofrezca productos o servicios, hoy día tiene como prioridad la constante evaluación de sus estrategias empresariales en pro de una progresiva adaptación que satisfaga los requerimiento y necesidades de sus clientes o consumidores.

Para Guiu (2012), las compañías que se desarrollan en medio de la globalización y una creciente competencia, están llamadas a fidelizar a sus clientes debido a que, sólo contar con clientes satisfechos es insuficiente, y por lo tanto es imprescindible conseguir su lealtad. Afirma que para ello es necesario colocar al cliente en el centro del negocio y dejar de un lado la tendencia general de las empresas de fortalecer las actividades de captación frente a las herramientas de fidelización. De la misma manera agrega que "expertos aseguran que la mejor forma de conseguir la lealtad de un cliente es ofreciéndole, además de un buen producto, la mejor experiencia de usuario”. (párr.1, 3)

De la misma idea puede inferirse que, el mercado mundial cuenta básicamente con dos tipos de usuarios o consumidores que serían, los que buscan productos más especializados y complejos y los que se conforman con cubrir sus necesidades de forma simple y barata, y ello es producto de los momentos de crisis que se han generado. Explica que, "hay que analizar el ciclo de vida de cada uno y actuar de forma consecuente y diferenciada en los casos en los que se detecten quejas o posibles bajas" pues "una buena gestión de las quejas convierte a los clientes descontentos en portavoces de la marca" (párr.4)

A continuación, se estima desarrollar la idea que algunos entendidos en la materia tienen sobre la fidelización de clientes, su importancia, exponer sobre algunas de las formas de identificación del tipo de cliente previo al desarrollo de estrategias de fidelización y finamente se expondrá sobre el Inbound marketing.

\section{Materiales y Métodos}

En esta investigación se ha dispuesto un determinado material bibliográfico compuesto por libros, revistas especializadas, trabajos de grado y postgrado, artículos científicos y demás publicaciones tanto físico como en versión digitalizada, que fueron escogidas a criterio del equipo investigador y entorno al tema planteado. 
El presente material se considera un compendio bibliográfico con el que se pretende exponer sobre algunos métodos de fidelización generalmente más utilizados en la actualidad, haciendo especial énfasis en la descripción de la estrategia conocida como: Inbound marketing, esto es con la finalidad de que el presente material pueda ser aprovechado como fuente de ampliación conocimiento, tanto para especialistas en la materia como para futuros autores de ulteriores trabajos investigativos.

En base a tales fines se ha llevado a cabo una investigación de naturaleza documental y de tipo descriptivo, extrayendo y analizando la información de las fuentes bibliográficas antes nombradas, teniendo como principio la consideración de criterios de actualidad y veracidad del contenido seleccionado.

Se afirma que la presente investigación responde a una metodología documental en base a lo entendido de autores como Ávila (2006), quien, en su obra, interpretando a otros autores, logra definir a la investigación documental como "una técnica que permite obtener documentos nuevos en los que es posible describir, explicar, analizar, comparar, criticar entre otras actividades intelectuales, un tema o asunto mediante el análisis de fuentes de información” (p. 50).

En cuanto a la profundidad de la investigación, se considera que bastaría con hacer mención de explicación hecha por Ríos (2017) quien al respecto afirma que una investigación se considerada a nivel descriptivo cuando “...busca encontrar las características, comportamiento y propiedades del objeto de estudio, ya sea en el presente o en el futuro..." (p.81).

\section{Resultados}

En base a lo discernido por Cancho, Govea, \& Sánchez (2017) logran resumir, entre otras cosas que:

En la fidelización se busca que las empresas se consoliden y permanezcan con el tiempo, la fidelidad se crea cuando se forma un vínculo entre el cliente y el servidor o proveedor del servicio de tal manera que ambas partes logran quedar satisfechas, muchas veces los clientes se vuelven asiduos y podrían recomendar el producto o servicio, este sería un punto favorable para continuar captando clientes y con el tiempo ir logrando que más clientes se fidelicen. (pág.26).

Así mismo Cancho et al. (2017) definen a la fidelización como "un lazo que se establece entre el cliente y la empresa" procurando que éste pueda sostenerse durante un largo período o lapso 
indeterminado. Agregan que "es básicamente la lealtad y compromiso entre ambos tanto como para el cliente interno como el externo para estrechar vínculos prolongados”. (pág.25-26)

Ejjaberi (2018) cita el trabajo de (Cronin, Brady y Hult, 2000) para indicar que "La satisfacción que percibe el cliente es el reflejo de la calidad del producto o servicio que ofrecen las empresas." Más adelante se refiere a ellos nuevamente para agregar que "El concepto de satisfacción en todas las áreas de estudio deviene importante ya que, si los clientes sienten satisfacción por una marca o producto, realizarán con mayor probabilidad compras repetitivas y futuras recomendaciones del mismo" (pág.3).

Hay que tener claro lo confirmado por especialistas tales como Fernández (2014), quien, respecto a la fidelización de clientes, asegura que es más barato fidelizar a los clientes que ya tienes. Explica que, en la actualidad, conseguir nuevos clientes implica incurrir en gastos considerables, y pocos ecommerce pueden permitirse ese desembolso económico. Agrega que, entre otros, esos gastos podrían ser de: impresiones, anuncios, clics, SEO y SEM.

Respecto a estos dos últimos se hace necesario hacer referencia a la explicación aportada por Canadell (2018), quien dice que SEO son las siglas de Search Engine Optimization (optimización para motores de búsqueda) y está referido a "los resultados orgánicos, esto es, que no vamos a pagarle a la empresa del buscador para que nuestras páginas posicionen mejor"; y SEM vienen siendo las siglas de Search Engine Marketing (marketing en motores de búsqueda) y se refiere a "las técnicas que mejoran el posicionamiento de nuestra web a través de anuncios pagados que aparecen en los buscadores para determinadas palabras clave" y agrega que "el SEM engloba todas las técnicas usadas para mejorar el posicionamiento de una web (incluyendo el SEO)" (párr.1-2, 67).

Herrero (2013) indica que "conseguir un cliente no es sencillo, hacer que repita y vuelva a comprar, lo es menos aún, por tanto, hay el empresario debe buscar siempre fórmulas que le ayuden a lograr clientes fieles". Así mismo ha confirmado que desde años atrás, la internet se convirtió en un medio generador de nuevos puntos de encuentro entre clientes y empresas, ya que este fenómeno ha propiciado un cambio de rutinas en las personas, haciendo que estas pasen más tiempo conectados a la red, ya sea desde una computadora personal o desde sus dispositivos móviles, lo que se traduce en un espacio de oportunidad para que las empresas "para dejar satisfechos a sus clientes e invitarles a repetir la experiencia de compra”. (párr..1-2). 


\section{Importancia de la fidelización del cliente}

De acuerdo a instituciones especializadas en el área como (EAE Business School, s.f.) la importancia radica en que sus aplicaciones "implica diferenciarse de la competencia, escuchar al cliente, ofrecer novedades, mejoras y, en suma, revierten en un mejor servicio" y con eso se presume que se logra "no sólo una manera de retener a antiguos clientes sino también a los nuevos, así como de atraer a clientes potenciales". Agregan que la fidelización del cliente es necesaria debido a auge competitivo entre empresas, además de que trasciende "el hecho de que el número de clientes potenciales acabará estancándose y la retención será obligatoria”. Afirman que, por un lado, la fidelización "permite hacer crecer el negocio, pero por otro puede ser de gran ayudar para mantener ingresos fijos que nos ayuden a la subsistencia del negocio". Finalmente indican que cuando se ejecutan las estrategias de fidelización inteligentes, se traducen en "rentabilidad y en valor empresarial". (párr.3)

\section{Conociendo al cliente antes de desarrollar estrategias de fidelización}

Respecto a este asunto cabe hacer mención a Sánchez (2017), quien ofrece un destacado aporte al explicar cómo conocer a los clientes a quienes se estima dirigir las estrategias de fidelización, a fines de que tales acciones puedan ser ajustadas en relación a importantes variables tales como: gustos, gastos medios, frecuencia de compra, entre otros, que en definitiva ayudaran en la toma de decisiones. Entre las distintas formas refiere:

A través del personal de venta: pues los vendedores y comerciales están en constante comunicación con nuestros clientes y ellos pueden conseguir información valiosa solo con escuchar sus preferencias, gustos y hacer algunas preguntas para mejorar los servicios.

Quejas: debido a que por medio de la resolución de estos problemas se pueden mejorar futuros procesos de ventas. Las quejas permiten conocer las inconformidades de nuestros clientes y mejorar a su vez el servicio. Darle valor es muy importante, ya que muchos más clientes pueden tener el mismo problema y simplemente en vez de quejarse, dejar de comprarnos y pasar a la competencia directamente.

Ver redes sociales: puesto que es muy importante saber quién nos sigue en las redes, cuál es su comportamiento, qué le gusta, qué comenta y a quién sigue dice mucho de cada persona, y es tarea de los directores de una organización unir toda la información disponible y sacar partido de ella. 
Encuestas: ya que las opiniones de todos los que de una manera u otra interactúan con la empresa, son importantes. Deben ser cortas y con opciones de respuesta, facilitando todo el trabajo al cliente y que tarde lo mínimo posible en realizarlas, de lo contrario, seguramente las ignorará.

Análisis con Google Analytics: esto es en caso de que se posea una página web. Por medio de esta herramienta se puede ver quienes entran en la página, desde qué equipo lo hace, desde que lugar y su comportamiento en la página. Así mismo puede averiguarse qué paginas dentro de tu web interesan más (dónde pasan más tiempo los internautas), cuál fue la primera pestaña que abrieron, y cuál fue la última. Si se tiene negocio online, puede verse más detalladamente qué compraron, o en qué parte del proceso de compra abandonaron

Mapa de empatía: esta herramienta es utilizada por las empresas para acercarse a los pensamientos y sentimientos que tiene el cliente poniéndose en la piel de ellos.

Ahora bien, considerando que hasta aquí se ha contextualizado la idea general de la fidelización de clientes, se puede comenzar a describir las distintas herramientas que en la actualidad son usadas como estrategias de fidelización en cualquier empresa que tiende a desenvolverse en un ambiente globalizado.

\section{Estrategias de fidelización}

En el trabajo de Pacheco (2018), se encuentran algunas de las estrategias más usadas para la fidelización de clientes, estas son:

\section{Personalización de la oferta}

Tras el del análisis de los hábitos de compra y consumo de los clientes, hay que tratar de "hacerle llegar información sobre aquellos productos nuevos en el catálogo que le pueden interesar o personaliza el contenido de tus campañas de e-mail marketing haciendo que se sienta único”. Dejando de un lado al producto como tal y centrándose en el proceso de compra es posible valerse “de las herramientas tecnológicas para ofrecer valor añadido y diferenciar a tu cliente fiel del cliente esporádico con opciones como sistemas de autogestión de pedidos, navegación por una web sin publicidad, canales alternativos de comunicación”. (p.30). 


\section{Creación de comunidad social y sentido de pertenencia}

El autor explica que los conocidos Social Media "dan la oportunidad de practicar un marketing relacional directo" con el cliente más leal. De allí, la idea es aprovechar "las redes sociales para generar contenido" del producto y propiciar, no sólo que hablen del mismo, sino que interactúen con él, comentándolo, compartiéndolo. (p.31)

\section{Procurar el seguimiento del intercambio}

Esta atención al cliente, en el punto anterior no se ciñe al proceso de compra y pago, sino que va más allá, si lo que se pretende en salvaguardar la lealtad del consumidor. Es más que conveniente, traspasado un breve tiempo desde la adquisición del producto o servicio, se debe interesar por el grado de satisfacción del cliente. Los llamados sistemas feedback, es decir la retroalimentación del proceso, sirven para mejorar el servicio que presta.

Si hubiera alguna queja justificada por parte de usuario se debe asumir la responsabilidad y facilitar ayuda. Hacer del problema una oportunidad y enseñar cómo se debe actuar en caso de mala experiencia. (p.31)

\section{Premiar la fidelidad}

El grado de satisfacción con el cliente más fiel surge cuando se ofrece ofertas, promociones, concursos y en definitiva mejores condiciones de compra. Son técnicas tradicionales, pero útiles a la hora de fortalecer la lealtad con el usuario, hacerlo sentir único y cuidado e incentivar las ventas del negocio. (p.32)

\section{Enviar newsletters (Boletines) periódicamente}

Ofrecer una oferta personalizada y hacérsela llegar al cliente mediante newsletters permite segmentar en función del contenido y el destinatario.

La práctica del e-mail marketing aportará valor añadido a tu campaña de fidelización. Además, la práctica del envío de newsletter propiciará el crecimiento de tu base de datos y las ventas cruzadas. (p.32)

Ahora bien, se ha considerado importante reiterar la obra de Herrero (2013), quien basándose en el modelo de gestión lean; que responde a tres pilares fundamentales conocidos como: aportación de valor al cliente, eliminación del despilfarro y desarrollar procesos flexibles, ha aportado la 
descripción de cinco (05) estrategias de fidelización que especialmente guardan relación con gran parte del objetivo propuesto inicialmente. Dicho especialista ejemplifica:

\section{El aporte de valor al cliente generando contenido de calidad}

El autor explica que "Internet es un canal en el que las personas utilizan diversos servicios, muchos de ellos relacionados con el consumo de contenido." Indica que, por ejemplo, se puede hacer un "blog de temática relacionada con los productos y servicios que ofrece a sus clientes, o un foro con temática similar y contenido aportado por la empresa y sus clientes o fans, así como otros formatos similares", y aclara que esto debe responder a un factor común, que no es más que dicho "contenido sea de calidad y actúe como polo de atracción de la atención de los clientes actuales y potenciales"

\section{El aporte de valor al cliente conversando con él de manera sencilla}

Se refiere a que la empresa ofrezca a sus clientes "canales de contacto eficientes, que gestionan correctamente los problemas, dudas y peticiones de información que este pueda plantear" aprovechando los distintos servicios online (chat, email, redes sociales, foros, blogs, entre otros. Explica que la idea no es la empresa abra multitud de cuentas en cuanto sitio que inmediatamente se ponga de moda al menos un par de días, sino en analizar la oferta de estas posibilidades en el mercado "para detectar por donde se mueven los clientes, abriendo un canal bidireccional en aquellos servicios en los que haya presencia suficiente de clientes y que además el formato lo permita"

\section{El aporte de valor al cliente mostrándole que hay vida al otro lado de la pantalla}

Indica que la atención de calidad es una de las estrategias que ayuda a generar confianza para que un cliente repita su experiencia de compra y especialmente, a través de Internet, puede sentir que "se enfrenta al sistema que ve en la pantalla y nada más, o bien, de que tras la pantalla hay un sistema sólido y que alguien vela por que todo el proceso de compra resulte satisfactorio”.

Refiere que en el comercio electrónico se den asumir ciertos riesgos y uno de ellos es que los sistemas simplemente fallan, pero esa eventualidad puede transformarse en una "oportunidad enorme si la empresa resuelve con eficacia una situación de este tipo". Asegura que "un cliente que ve que algo falla en un proceso pero que la empresa responde, no tendrá reparo en volver porque sabe que con ella sus expectativas están cubiertas incluso cuando algo no sale bien”. 


\section{Eliminando el despilfarro que afecta al cliente}

El autor asegura que es bastante frecuente que el cliente termine siendo afectado por algo que no está funcionando como debería ser, debido a las propias ineficiencias que surgen en los procesos de un negocio. Con un ejemplo explica que "un despilfarro que afecta a un cliente es el de los call centers mal gestionados, que obligan a perder el valioso tiempo de los clientes cuando tienen que reclamar o pedir información sobre algo relacionado con la empresa". Sin embargo, respecto al mismo ejemplo, aclara que un sistema de ayuda al cliente vía Internet podría mejorar la experiencia

\section{Desarrollando procesos flexibles que se adapten al cliente}

Ofrecer al cliente la posibilidad de acceder desde cualquiera de sus dispositivos es una vía de fidelización, dado que supone trabajar con procesos flexibles, que se adaptan a las diferentes vías de acceso que utiliza como usuario, siendo él quien elige con cual accede.

Enfatiza diciendo que, por ejemplo:

Una tienda online que además de acceso vía navegadores para equipos de escritorio sea compatible con los de smartphone o tabletas, estará poniendo a disposición de sus clientes un proceso de compra más flexible y, por tanto, tendrá más posibilidades de que estos completen el circuito de compra, de que recomienden sus servicios y de que retornen en el futuro para volver a comprar

\section{El Inbound marketing}

Para Tomas (2018) la idea de esta estrategia se centra en atraer a los clientes para que dejen sus datos de contacto y se conviertan en un lead, generando así la posibilidad de empezar a alimentar una relación con estos. Refiere que entre las estrategias más comunes para alcanzar dicho objetivo está la de creación de contenido de valor que resuelva los problemas reales de los clientes potenciales, que bien puede facilitarse mediante la elaboración de artículos de blog, vídeos, ebooks, informes y otros contenidos. (párr.26-27)

Viteri (2011) dice que "Es una nueva manera de entender el marketing. Consiste en generar prospectos, y trabajar con ellos para convertirlos en clientes". Continúa explicando que:

El centro del Inbound marketing radica en los contenidos. Cuando ya esté creado el contenido (videos, documentos, presentaciones, eventos, etc.), la clave está en socializarla por medio de la 
Web 2.0. Estas técnicas son conocidas como Social Media Marketing, SEO Marketing, y "Blogging", las cuales resultan fundamentales para obtener resultados en este nuevo paradigma.

El Inbound Marketing es todo aquello que interacciona con el cliente de tal forma que este tiene la iniciativa, lo cual aporta algo muy valioso las empresas: su atención y predisposición.

El Inbound marketing es una nueva estrategia de marketing para negocios que se enfoca en las cambiantes tendencias de negocio y el uso de las ultimas herramientas y técnicas de la Web 2.0 para anunciar productos y servicios, y extenderse hacia los clientes. (p.30-31).

\section{Conclusiones}

Las empresas deben invertir en nuevas tecnologías como herramientas de fidelización, y aprovechar las soluciones tecnológicas que permiten cada vez más mejorar la comunicación entre cliente y empresa, por medio de la versatilidad de canales disponibles tales como: teléfonos, portales web, aplicaciones especializadas para los distintos dispositivos móviles, sin embargo, hay que tener siempre presente la adaptación de dichos canales al tipo de cliente que se posee, porque a fin de cuentas éstos son quienes deben determinar cómo quieren que se comuniquen con ellos, pues de lo contrario, la experiencia será frustrante. (Guiu, 2012)

El Inbound marketing podría ser considerado en la actualidad como una excelente alternativa en comparación con otros métodos de fidelización de clientes ya que, que, dependiendo del caso, los otros llegan a concebirse más complejos y costoso de ejecutar, y otros no tan efectivos en la actualidad.

\section{Referencias Bibliográficas}

Ávila, H. (2006). Introducción a la metodología de investigación. Ciudad de Cuauhtemoc Chihuahua - México: Edición Electrónica.

Canadell, M. (16 de 04 de 2018). cyberclick.es. Recuperado el 31 de 07 de 2018, de cyberclick.es: https://www.cyberclick.es/numerical-blog/que-es-seo-sem-diferencias-significado- $\quad$ y-usocombinado

Cancho, C., Govea, K., \& Sánchez, G. (08 de 2017). repositorio.ulasamericas.edu.pe. Recuperado el 31 de 07 de 2018, de repositorio.ulasamericas.edu.pe: http://repositorio.ulasamericas.edu.pe/bitstream/handle/upa/257/LA\%20CALIDAD\%20DEL\%20 SERVICIO\%20Y\%20FIDELIZACI\%C3\%93N\%20DEL\%20CLIENTE\%20EN\%20LA\%20AGE 
NCIA\%20PRINCIPAL\%20DE\%20LA\%20CAJA\%20METROPOLITANA\%2c\%20LIMA\%202 016.pdf? sequence $=1 \&$ is Allowed $=y$

EAE Business School. (s.f.). eaeprogramas.es. Recuperado el 31 de 07 de 2018, de eaeprogramas.es: https://www.eaeprogramas.es/empresa-familiar/que-es-la-fidelizacion-declientes-y-por-que-es-importante.

Ávila, H. (2006). Introducción a la metodología de investigación. Ciudad de Cuauhtemoc Chihuahua - México: Edición Electrónica.

Canadell, M. (16 de 04 de 2018). cyberclick.es. Recuperado el 31 de 07 de 2018, de cyberclick.es: https://www.cyberclick.es/numerical-blog/que-es-seo-sem-diferencias-significado- $\quad$ y-usocombinado

Cancho, C., Govea, K., \& Sánchez, G. (08 de 2017). repositorio.ulasamericas.edu.pe. Recuperado el 31 de 07 de 2018, de repositorio.ulasamericas.edu.pe: http://repositorio.ulasamericas.edu.pe/bitstream/handle/upa/257/LA\%20CALIDAD\%20DEL\%20 SERVICIO\%20Y\%20FIDELIZACI\%C3\%93N\%20DEL\%20CLIENTE\%20EN\%20LA\%20AGE NCIA\%20PRINCIPAL\%20DE\%20LA\%20CAJA\%20METROPOLITANA\%2c\%20LIMA\%202 016.pdf? sequence $=1 \&$ is Allowed $=\mathrm{y}$

EAE Business School. (s.f.). eaeprogramas.es. Recuperado el 31 de 07 de 2018, de eaeprogramas.es: https://www.eaeprogramas.es/empresa-familiar/que-es-la-fidelizacion-declientes-y-por-que-es-importante

Ejjaberi, A. E. (02 de 2018). http://diposit.ub.edu. Recuperado el 31 de 07 de 2018, de http://diposit.ub.edu: http://diposit.ub.edu/dspace/bitstream/2445/122649/1/AEE_TESIS.pdf Fernández, M. (05 de 11 de 2014). lancetalent.com. Recuperado el 31 de 07 de 2018, de lancetalent.com: https://www.lancetalent.com/blog/5-estrategias-infalibles-fidelizar-clientesecommerce/

Guiu, D. (2012). socialetic.com. Recuperado el 31 de 07 de 2018, de socialetic.com: https://www.socialetic.com/claves-fidelizacion-clientes-empresas-de-servicios.html

Herrero, P. (2013). sage.com. Recuperado el 31 de 07 de 2018, de sage.com: https://www.sage.com/es-es/blog/estrategias-de-fidelizacion-de-clientes-a-traves-de-internet/\# Pacheco, R. (2018). http://repositorio.ulvr.edu.ec. Recuperado el 31 de 07 de 2018, de http://repositorio.ulvr.edu.ec/: http://repositorio.ulvr.edu.ec/bitstream/44000/2305/1/T-ULVR2102.pdf 
Ríos, R. (2017). eumed.net. Recuperado el 31 de 07 de 2018, de eumed.net: http://www.eumed.net/libros/img/portadas/1662.pdf

Sánchez, S. (16 de 05 de 2017). puromarketing.com. Recuperado el 31 de 07 de 2018, de puromarketing.com: https://www.puromarketing.com/14/28784/fidelizacion-clientes.html Tomas, D. (13 de 04 de 2018). cyberclick.es. Recuperado el 31 de 07 de 2018, de cyberclick.es: https://www.cyberclick.es/numerical-blog/6-estrategias-para-captar-y-fidelizar-clientes Viteri, J. (2011). bibdigital.epn.edu.ec. Recuperado el 31 de 07 de 2018, de bibdigital.epn.edu.ec: http://bibdigital.epn.edu.ec/bitstream/15000/7711/1/CD-4373.pdf. 